\title{
Finanzielle Kompetenzen und Defizite in Deutschland - eine aktuelle Bestandsaufnahme
}

\author{
Ronald Bachmann $(\mathbb{D} \cdot$ Christian Rulff $\cdot$ Christoph M. Schmidt
}

Angenommen: 8. Oktober 2021 / Online publiziert: 29. Oktober 2021

(C) Der/die Autor(en) 2021

Zusammenfassung Die Anforderungen an die Finanzkompetenzen der Bevölkerung steigen aus einer Reihe von Gründen weiter an. Anhand des Datensatzes „Private Haushalte und ihre Finanzen“ zeigt diese Studie, dass in Deutschland generell ein relativ hohes Niveau an Finanzkompetenz vorliegt; dennoch besteht hier Verbesserungspotenzial. Dies gilt insbesondere für bestimmte sozio-demographische Gruppen wie Frauen, Personen mit Migrationshintergrund sowie finanzschwache Haushalte. Hieraus ergibt sich Handlungsbedarf, der für verschiedene Felder diskutiert wird. Es wird insbesondere empfohlen, Finanzbildung stärker in die Lehrpläne von Schulen zu integrieren, die Ausbildung von Lehrern in diesem Bereich zu verbessern, Bildungs- und Informationsangebote für Erwachsene zu stärken und die Transparenz von Finanz- und Versicherungsprodukten zu erhöhen.

Schlüsselwörter Finanzielle Kompetenz $\cdot$ Rentenplanung $\cdot$ Haushaltsvermögen

Ronald Bachmann $(\bowtie) \cdot$ Christian Rulff · Christoph M. Schmidt RWI - Leibniz-Institut für Wirtschaftsforschung e. V., Hohenzollernstr. 1-3, 45141 Essen,

Deutschland

E-Mail: bachmann@rwi-essen.de

Ronald Bachmann

Düsseldorf Institute for Competition Economics (DICE), Heinrich-Heine-Universität Düsseldorf, Universitätsstr. 1, 40225 Düsseldorf, Deutschland

Ronald Bachmann · Christoph M. Schmidt

Forschungsinstitut zur Zukunft der Arbeit GmbH (IZA), Schaumburg-Lippe-Str. 5-9, 53113 Bonn, Deutschland

Christian Rulff

Fakultät für Wirtschaftswissenschaften, Universität Duisburg-Essen, Universitätsstr. 12, 45141 Essen, Deutschland

Christoph M. Schmidt

Fakultät für Wirtschaftswissenschaft, Ruhr-Universität Bochum, Universitätsstr. 150, 44801 Bochum, Deutschland 
JEL Klassifikation G53 · A20 · D14

\title{
Financial competencies and deficits in Germany-a current review
}

\begin{abstract}
The requirements for financial literacy among the population continue to rise for a number of reasons. Using the dataset "Panel on Household Finances", this study shows that Germany generally has a relatively high level of financial literacy; nevertheless, there is room for improvement here. This is particularly true for certain socio-demographic groups such as women, people with an immigrant background and financially weak households. This gives rise to a need for action, which is discussed for various fields. In particular, it is recommended to integrate financial education more strongly into school curricula, to improve teacher training in this area, to strengthen educational and information offerings for adults and to increase the transparency of financial and insurance products.
\end{abstract}

Keywords Financial literacy $\cdot$ Retirement planning $\cdot$ Household wealth

\section{Einleitung}

Haushalte sind immer mehr gefordert, komplexe Sachverhalte zu verstehen, um fundierte Entscheidungen treffen zu können, die für ihr Leben von erheblicher finanzieller Tragweite sind. Dies kann ohne finanzielle Grundkompetenzen nicht oder nur unter bisweilen erheblichen Einbußen bei den persönlichen Handlungsspielräumen gelingen. So hat sich in den vergangenen Jahren besonders in westlichen Industrienationen ein Trend entwickelt, Dinge des täglichen Gebrauchs nicht mehr zu kaufen, sondern Zugang zu Konsumgütern im Rahmen von Nutzungsverträgen zu erwerben. Wichtige Beispiele sind Verträge zur Nutzung von Mobiltelefonen, Musik- und Videodienstleistern sowie Sportangeboten. Autos werden mittlerweile typischerweise geleast oder, falls sie erworben werden, über längere Zeiträume finanziert.

Haushalte wenden zudem einen erheblichen Anteil ihres Einkommens für Versicherungen und private Altersvorsorge auf - besonders dabei ist grundlegendes Finanzwissen für fundierte Entscheidungen unerlässlich, da die Wahl zwischen verschiedenen komplexen Finanzprodukten meist besonders weitreichende Konsequenzen hat. Die zur Verfügung stehenden Finanzprodukte haben sich im Laufe der Zeit immer weiter verändert und sind teilweise deutlich komplexer geworden (Lusardi 2019; Lusardi und Mitchell 2014). Insbesondere angesichts der sich abzeichnenden Rentenlücke für die derzeitigen Erwerbstätigen und der andauernden Niedrigzinsphase, die gepaart mit der wenig Aktien-affinen Anlagestrategie der meisten Deutschen zu einer realen Entwertung ihres Vermögens führt, ist ein fundiertes Wissen über Finanzen für Privatinvestoren wichtiger denn je.

Im Einklang mit dieser Einschätzung legen empirische Studien in der Tat nahe, dass ein hohes Niveau an finanziellen Grundkompetenzen eine Vielzahl positiver Auswirkungen hat. So weisen Personen mit einem hohen Grad an finanziellen Grundkompetenzen in der Regel ein höheres Nettovermögen auf, planen häufiger für die Rente, partizipieren mit einer höheren Wahrscheinlichkeit am Finanzmarkt 
und treffen im allgemeinen fundiertere finanzielle Entscheidungen. Zwar schneidet Deutschland beim Thema Finanzkompetenz im internationalen Vergleich in der Regel gut ab, jedoch finden sich auch hier teilweise sehr große Unterschiede über verschiedene sozio-demographische Gruppen hinweg (Bucher-Koenen und Lusardi 2011; Schmidt und Tzamourani 2017; Stolper und Walter 2017).

Trotz dieser hohen und stetig wachsenden Bedeutung der finanziellen Grundkompetenzen für die individuelle Wohlfahrt genießt das Thema in Wissenschaft und Politik nach wie vor eine relativ geringe Aufmerksamkeit. Um eine fundierte Basis für diesen Diskurs zu schaffen, analysiert das vorliegende Papier für Deutschland, wie hoch aktuell das Niveau an finanziellen Grundkompetenzen in der Bevölkerung ist, welche Gruppen im Durchschnitt das höchste Risiko aufweisen, über (zu) geringe finanzielle Grundkompetenzen zu verfügen, und wie sich die finanziellen Grundkompetenzen zwischen verschiedenen Regionen Deutschlands unterscheiden. Datengrundlage der empirischen Analyse ist der Datensatz „Private Haushalte und ihre Finanzen“ (PHF) der Deutschen Bundesbank.

Das Papier geht dabei wie folgt vor. Zunächst wird in Abschn. 2 auf die Messung von finanziellen Grundkompetenzen eingegangen, die für Deutschland zur Verfügung stehenden Datensätze vorgestellt und die aus der Literatur gewonnenen Ergebnisse kurz zusammengefasst. In Abschn. 3 werden deskriptive Ergebnisse zur Verteilung der finanziellen Bildung in Deutschland im internationalen Kontext und die finanzielle Bildung über verschiedene sozio-demographische Charakteristika sowie Regionen vorgestellt. In Abschn. 4 werden wirtschafts- und bildungspolitische Schlussfolgerungen gezogen und Abschn. 5 fasst die Ergebnisse noch einmal zusammen.

\section{Die Messung finanzieller Grundkompetenzen}

Finanzielle Bildung wird in der Literatur in der Regel näherungsweise anhand von strukturierten Befragungen gemessen, in denen bestimmtes Wissen über einzelne Finanzbereiche abgeprüft wird. Die drei gängigsten Fragen wurden von Lusardi und Mitchell (2008) für die Studie „Health and Retirement“ im Jahr 2004 eingeführt. In der Literatur werden diese Fragen als die „big three“ bezeichnet, da sie seit ihrer Einführung die Standardfragen bei Befragungen zum Thema Finanzkompetenzen darstellen. Diese „big three“ sprechen verschiedene Themen aus dem Finanzbereich an: das Wissen über Zinsen, das Wissen über Inflation und das Wissen über die Risikoeinschätzung von verschiedenen Finanzprodukten. ${ }^{1}$

Während Fragen zu den ersten beiden Themen im Prinzip auch mit grundlegenden Rechenkenntnissen beantwortet werden können, wird bei der dritten Frage zumindest eine gewisse Kenntnis über die Funktionsweise von Finanzmärkten vorausgesetzt. Aus diesem Grund werden die Inhalte der ersten beiden Fragen häufig als finanzielle Grundkenntnisse bezeichnet, während der Inhalt der dritten Frage zu den fortgeschrittenen Finanzkompetenzen zählt. Obwohl Teilnehmende mit besseren Rechenkenntnissen und kognitiven Fähigkeiten die „big three“ besser beantworten,

\footnotetext{
1 Der Anhang enthält den genauen Wortlaut der Fragen.
} 
erklärt dies nicht die gesamte Variation der Finanzkompetenzen (Lusardi et al. 2010), so dass die „big three“ grundsätzlich als ein sinnvolles Maß für die Messung finanzieller Kompetenzen angesehen werden.

Nichtsdestotrotz ist zu beachten, dass die auf Basis der „big three“ ermittelten Erkenntnisse gewisse Limitationen aufweisen. So hängen die Antworten der Teilnehmenden stark von der genauen Formulierung der Frage ab (Lusardi und Mitchell 2011; Van Rooij et al. 2011). Außerdem gibt es für die Teilnehmenden keine Anreize, die Antworten korrekt und bedacht zu beantworten oder sich - wie im echten Leben - Unterstützung bei Familie, Freunden oder Finanzberatern zu suchen (Hastings et al. 2013). Daher wurden zu den „big three“ einige Alternativen entwickelt.

Eine auf den ersten Blick spannende alternative Möglichkeit, finanzielle Kompetenzen zu erheben, besteht in der Selbsteinschätzung: Hierbei werden die Befragten gebeten, selbst ihre Finanzkompetenz auf einer vorgegebenen Skala einzuschätzen. Die Validität dieser alternativen Vorgehensweise hat sich jedoch als zweifelhaft erwiesen. Vergleichsstudien zwischen der subjektiven Selbsteinschätzung und der gemessenen Finanzkompetenz haben gezeigt, dass die Befragten dazu tendieren, ihre Finanzkompetenz systematisch zu überschätzen (Bucher-Koenen et al. 2017).

Aus methodischer Sicht erscheint vielmehr ein Instrument zur Messung der Finanzkompetenz wünschenswert, dass aus einer Vielzahl an Fragen besteht und den Schwierigkeitsgrad dieser Fragen berücksichtigt. Dies ist insbesondere der Fall, weil gezeigt wurde, dass die „,big three“, ebenso wie eine um zwei zusätzliche Fragen erweiterte Version (,,big five“), aufgrund ihrer geringen Zahl an Fragen und der sehr groben Kategorisierung nur in eingeschränktem Maße geeignet sind, um ein tieferes Verständnis für die finanziellen Kompetenzen zu entwickeln (Nicolini und Haupt 2019). Knoll und Houts (2012) haben ein psychometrisch fundiertes Instrument zur Messung der Finanzkompetenz entwickelt, das aus 20 Fragen besteht, die unter anderem die „big three“ enthalten, und sich durch eine hohe Validität und Vergleichbarkeit auszeichnet. Knoll und Houts (2012) konnten für die USA zeigen, dass dieses Maß ein breiteres Spektrum an Finanzwissen erfasst und z.B. eine bessere Vorhersagekraft für die Frage besitzt, ob Individuen für die Rente planen. In Abwesenheit genauerer Maße stellen die „big three“ nichtsdestotrotz ein wichtiges $\mathrm{Ma} ß$ zur Messung der finanziellen Kompetenzen dar, sie weisen zudem eine hohe Korrelation mit dem Maß von Knoll und Houts (2012) auf.

Angesichts der Limitationen der verfügbaren Maße nutzt die Literatur teilweise soziodemografische Daten wie Einkommen, Vermögen, Alter, Geschlecht, Beruf, Intelligenz oder Bildungsstatus als Proxy für Finanzkompetenz (Bannier und Neubert 2016; Bianchi 2018; Christelis et al. 2010; Grinblatt et al. 2011; Lusardi und Mitchell 2011; Lusardi et al. 2010; Lusardi und Tufano 2015; Van Rooij et al. 2011). Andere Studien nutzen das beobachtete Ergebnis von finanziellen Entscheidungen als Maß für Finanzkompetenz; dies können beispielsweise die Risikodiversifizierung von Aktienportfolios oder vorherige Anlagekenntnisse sein (Goetzmann und Kumar 2008; Nicolosi et al. 2009; Seru et al. 2010).

Für Deutschland stehen zur direkten Messung der Finanzkompetenzen nur wenige Datensätze zur Verfügung. Hierzu zählt erstens die Studie ,,Sparen und Altersvorsorge in Deutschland“ (SAVE). Diese wurde jedoch im Jahr 2013 eingestellt und enthält lediglich für die Jahre 2007 und 2009 Fragen zur finanziellen Bildung (Börsch-Su- 
pan et al. 2008). Zweitens wurden in zwei Wellen der Innovationsstichprobe des Sozio-oekonomischen Panels ebenfalls Fragen zu den Finanzkompetenzen aufgenommen, die sich jedoch von den ,big three“-Fragen unterscheiden (Richter und Schupp 2015).

Drittens stehen die Daten der Studie „Private Haushalte und ihre Finanzen“ (PHF) zur Verfügung, die in diesem Beitrag genutzt werden. Diese Studie erhebt seit dem Jahr 2010 alle drei Jahre Daten zu Einkommen, Ausgaben und Vermögen privater Haushalte in Deutschland (Altmann et al. 2020). Bei der PHF-Studie handelt es sich um einen repräsentativen Paneldatensatz für deutsche Haushalte. Für die Analyse der vorliegenden Studie wurden die Daten aus den PHF-Studien der Jahre 2010/2011, 2014 und 2017 verwendet. Neben den Kernfragen zum Vermögen, der Verschuldung und dem Einkommen der Haushalte enthält der Datensatz ebenfalls Antworten auf die von Lusardi und Mitchell (2008) entwickelten „big three“-Fragen zur Erfassung der finanziellen Bildung.

\section{Finanzielle Grundkompetenzen in Deutschland}

Tab. 1 stellt die Ergebnisse zu den finanziellen Grundkompetenzen basierend auf allen verfügbaren Wellen des PHF-Datensatzes dar. Mit einem Anteil von $62 \%$ beantwortet im Vergleich zu Studien basierend auf den SAVE-Daten ein höherer Anteil der Befragten alle ,big three“-Fragen richtig. Dieser Unterschied kann zum Teil dadurch erklärt werden, dass die Fragen zur finanziellen Bildung im PHF nicht von allen Haushaltsmitgliedern, sondern lediglich von der für die Finanzen verantwortlichen Person beantwortet werden. Es ist wahrscheinlich, dass diese Personen über eine vergleichsweise hohe Finanzkompetenz verfügen. Typischerweise werden die beiden weniger voraussetzungsreichen Fragen zur finanziellen Grundbildung häufiger richtig beantwortet (Zinsen: $85 \%$ und Inflation: $89 \%$ ) als die Frage zur fortgeschrittenen finanziellen Bildung (Risikodiversifikation: $72 \%$ ). Somit scheinen

Tab. 1 Finanzkompetenzen im PHF. (Quelle: PHF 2010/2011, 2014, 2017. Eigene Berechnungen)

\begin{tabular}{ll}
\hline Frage 1 (Zinsen) & \\
Richtig beantwortet & $84,97 \%$ \\
Falsch beantwortet & $12,19 \%$ \\
Weiß nicht/keine Antwort & $2,84 \%$ \\
Frage 2 (Inflation) & \\
Richtig beantwortet & $88,64 \%$ \\
Falsch beantwortet & $8,28 \%$ \\
Weiß nicht/keine Antwort & $3,08 \%$ \\
Frage 3 (Risikodiversifikation) & \\
Richtig beantwortet & $72,21 \%$ \\
Falsch beantwortet & $13,90 \%$ \\
Weiß nicht/keine Antwort & $13,89 \%$ \\
Alle Fragen richtig & $61,70 \%$ \\
Beobachtungen & 12.700 \\
\hline
\end{tabular}


mathematische Kompetenzen besser ausgeprägt zu sein als Kenntnisse über finanzielle Mechanismen.

Andere Industrieländer weisen bei der finanziellen Bildung geringere Werte auf als Deutschland. So beantworten in Ländern wie der Schweiz, den Niederlanden sowie Australien zwischen $43 \%$ und $50 \%$ der Befragten alle drei ,big three“ richtig (Lusardi und Mitchell 2014; Alessie et al. 2011; Agnew et al. 2013; Brown und Graf 2013). Hierbei ist jedoch zu beachten, dass die Ergebnisse nicht ohne Weiteres untereinander verglichen werden können, da die Daten zu unterschiedlichen Zeitpunkten erhoben wurden, die Fragen von anderen Haushaltsmitgliedern beantwortet wurden und zum Teil leicht von den „big three“ abweichen. Anhand international vergleichbarer Ergebnisse, basierend auf der OECD/INFE 2020-Erhebung, weisen Personen aus Deutschland zwar leicht überdurchschnittliche Finanzkompetenzen auf, wenngleich sie absolut gesehen immer noch als relativ gering einzustufen sind. Dieses Ergebnis lässt sich auch dadurch erklären, dass die skandinavischen Länder, welche in anderen Umfragen in der Regel überdurchschnittliche Ergebnisse erzielen, an dieser Befragung nicht teilnehmen (OECD 2020).

In der Literatur werden eine Reihe von sozio-demographischen Charakteristika identifiziert, die mit finanzieller Bildung in Zusammenhang gebracht werden. Diese sind insbesondere das Geschlecht, Alter, Bildung, Migrationshintergrund, sowie finanzielle Faktoren (Einkommen/Vermögen). Die nachfolgenden Abschnitte gehen jeweils einzeln auf diese Faktoren ein. Dabei wird jeweils der aktuelle Forschungsstand anhand der einschlägigen Literatur vorgestellt und um aktuelle Ergebnisse für Deutschland anhand des PHF-Datensatzes ergänzt.

Die finanzielle Bildung unterscheidet sich stark zwischen Frauen und Männern. Dieses Ergebnis wird in einer langen Reihe von Studien über eine Vielzahl an Ländern dokumentiert und gilt anscheinend unabhängig vom sozioökonomischen oder kulturellen Hintergrund. In der Regel weisen Frauen eine geringere Wahrscheinlichkeit auf, die jeweilige Frage zur finanziellen Bildung korrekt zu beantworten. Darüber hinaus ist bei ihnen ebenfalls die Wahrscheinlichkeit höher, dass sie die Kategorie „weiß nicht“ wählen. Der Geschlechterunterschied ist nicht nur anhand der objektiven, gemessenen finanziellen Bildung zu beobachten, sondern gilt auch für die subjektive Selbsteinschätzung zur finanziellen Bildung (Lusardi und Mitchell 2014; Bucher-Koenen et al. 2017).

Eine mögliche Erklärung für diese Geschlechterunterschiede liefert z.B. Hsu (2016), wonach je nach gesellschaftlichem Kontext ein gewisser Grad an Unterschieden in finanzieller Bildung als rational betrachtet werden kann, da sich Partner innerhalb eines Haushaltes auf bestimmte Fähigkeiten spezialisieren. Dies erklärt jedoch nicht das geringere finanzielle Bildungsniveau von alleinlebenden Frauen, welche sich um ihre eigenen Finanzen kümmern müssen (Lusardi und Mitchell 2014).

Bucher-Koenen et al. (2017) untersuchen Geschlechterunterschiede in der finanziellen Bildung für die USA, die Niederlande und Deutschland und kommen zu dem Ergebnis, dass Frauen in allen drei Ländern eine geringere Wahrscheinlichkeit aufweisen, alle drei Fragen korrekt zu beantworten. In den USA beträgt dieser Unterschied 15,8 Prozentpunkte (Männer: 38,3\% vs. Frauen: 22,5\%), in den Niederlanden 22,1 Prozentpunkte (Männer: 55,1\% vs. Frauen: 35,0\%) und in Deutschland 


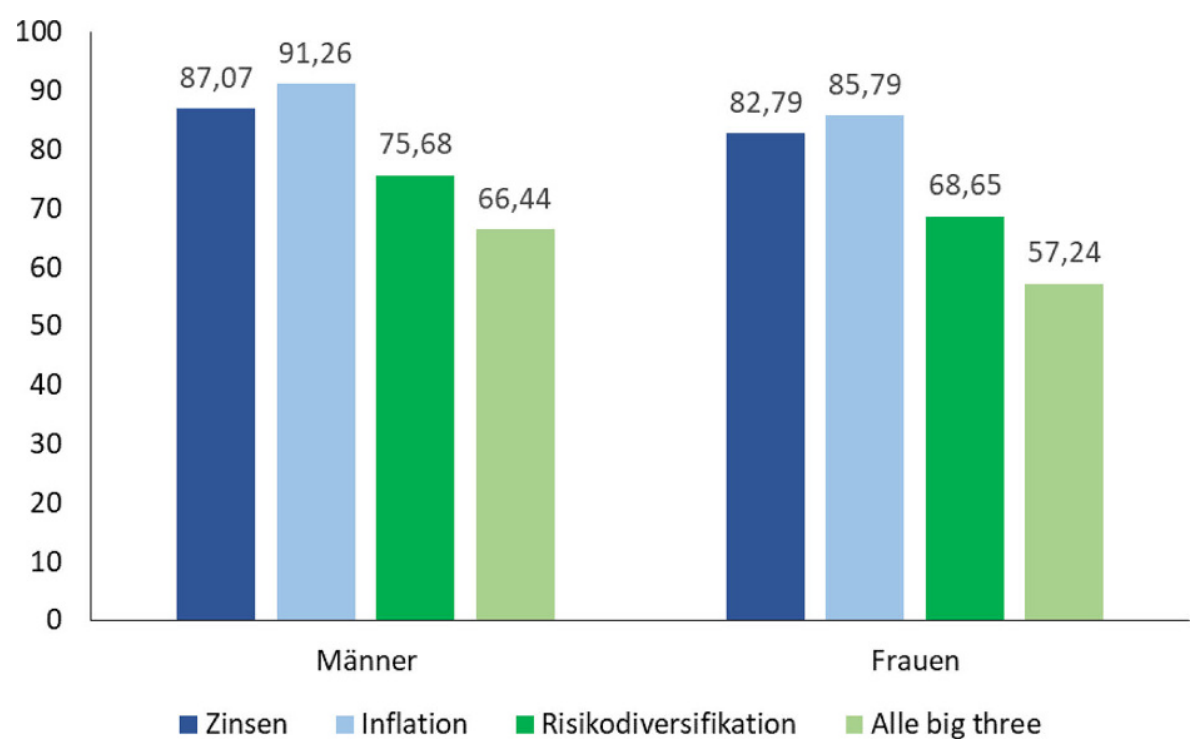

Abb. 1 Finanzkompetenzen nach Geschlecht. (Quelle: PHF 2010/2011, 2014, 2017. Eigene Berechnungen. Anmerkungen: Die Abbildung stellt den Anteil (in \%) an richtigen Antworten für die jeweilige Frage zur Finanzkompetenz, sowie den Anteil derer, die alle drei Fragen richtig beantwortet haben, dar)

12,1 Prozentpunkte (Männer: 59,5\% vs. Frauen: 47,5\%) (Bucher-Koenen et al. 2017). Für andere Länder wurden anhand derselben drei Fragen oder leichten Abweichungen hiervon in vergleichbaren Studien ähnliche Ergebnisse erzielt. ${ }^{2}$

Die Ergebnisse des PHF-Datensatzes für Deutschland stehen hiermit völlig im Einklang. In Abb. 1 ist zu erkennen, dass Frauen im Vergleich zu Männern einen geringeren Anteil an richtigen Antworten in allen drei Fragen erreichen. Die Zinsfrage beantworten $87 \%$ der Männer und $83 \%$ der Frauen richtig, die Frage zur Inflation wird von $91 \%$ der Männer und $85 \%$ der Frauen richtig beantwortet, und die Frage zur Risikodiversifikation wird von $76 \%$ der Männer und $68 \%$ der Frauen richtig beantwortet. Insgesamt beantworten $66 \%$ der Männer und $57 \%$ der Frauen alle drei Fragen richtig. Alle in Abb. 1 dargestellten Differenzen sind statistisch signifikant auf dem $0,1 \%$-Niveau.

Die finanzielle Bildung schwankt ebenfalls sehr stark über das Alter. Häufig wird dabei eine umgekehrte U-Form nachgewiesen, d.h. die finanziellen Kompetenzen sind bei Personen mittleren Alters besser ausgeprägt als bei jüngeren oder älteren Personen (Lusardi und Mitchell 2014). Dieser Zusammenhang zwischen Alter und finanzieller Bildung kann problematisch sein, da relativ junge Personen, welche am Anfang ihres Erwerbslebens stehen, häufig weitreichende finanzielle Entscheidungen treffen müssen, die über ihr finanzielles Wohlergehen in den kommenden Jahren entscheiden. Die geringe finanzielle Bildung bei der älteren Gruppe ist ebenfalls problematisch, da diese über einen Großteil des gesamten Vermögens einer

\footnotetext{
2 Siehe z.B. Agnew et al. (2013) für Australien, Arrondel et al. (2013) für Frankreich, Brown und Graf (2013) für die Schweiz und Sekita (2011) für Japan.
} 


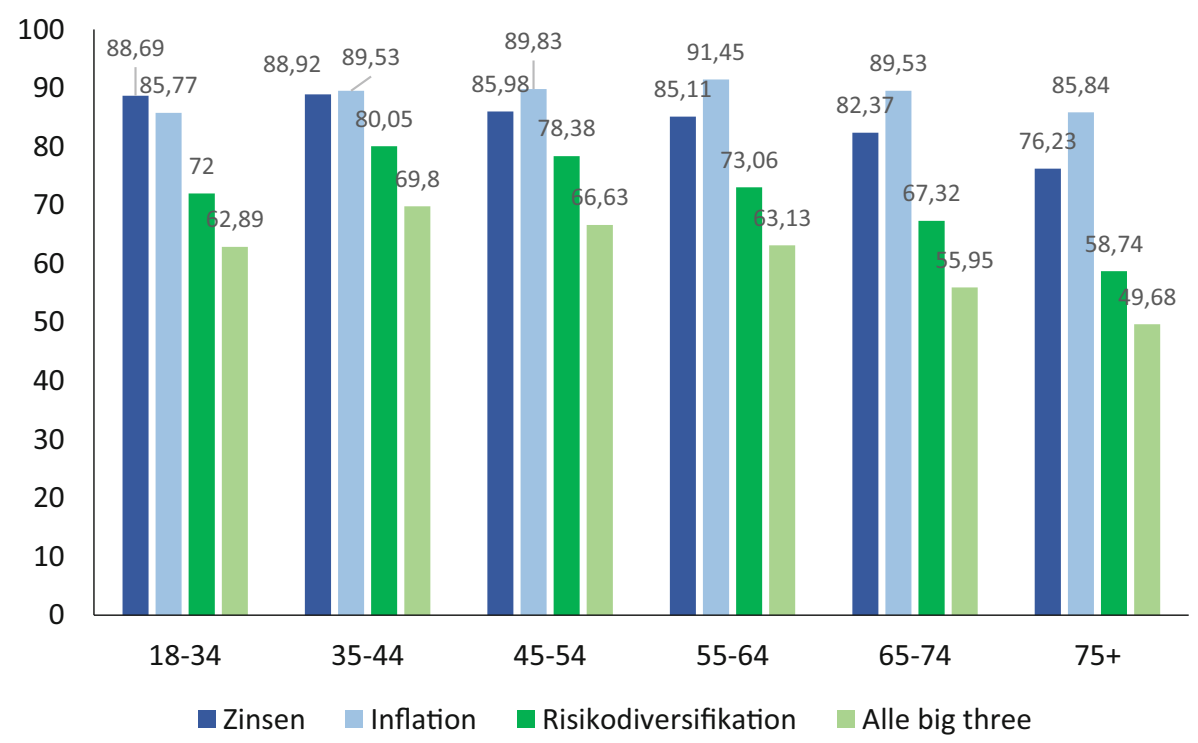

Abb. 2 Finanzkompetenzen nach Alter. (Quelle: PHF 2010/2011, 2014, 2017. Eigene Berechnungen. Anmerkungen: Die Abbildung stellt den Anteil (in \%) an richtigen Antworten für die jeweilige Frage zur Finanzkompetenz, sowie den Anteil derer, die alle drei Fragen richtig beantwortet haben, dar)

Volkswirtschaft verfügen und somit schlechtere Entscheidungen weitreichende gesellschaftliche Folgen haben können als bei anderen Altersgruppen (Stolper und Walter 2017).

Unklar ist bei dem beobachteten Verlauf jedoch, ob es sich tatsächlich um einen Alters- oder um einen Kohorteneffekt handelt. So haben Personen, die sich jetzt im Rentenalter befinden, geringere Erfahrungen mit modernen Finanzprodukten, da sie sich stärker auf die gesetzliche Rente verlassen konnten als Personen, die sich derzeit im Berufsleben befinden. Für frühere Kohorten war die Notwendigkeit, finanzielle Kompetenzen zu erlangen, daher deutlich geringer. Sie konnten sich auf hohe gesetzliche Rentenzahlungen in der Zukunft verlassen (Lusardi et al. 2017).

Auch in den PHF-Daten für Deutschland sind deutliche Unterschiede zwischen Altersgruppen zu erkennen. Abb. 2 zeigt die finanzielle Bildung der befragten Personen für die Altersgruppen der 18- bis 34-, 35- bis 44-, 45- bis 54-, 55- bis 64-, 65- bis 74- und der über 74-Jährigen. Bei der Frage zum Zinseffekt zeigen sich nur geringe Unterschiede zwischen den Altersgruppen. ${ }^{3}$ Die Gruppe der 35- bis 44Jährigen weist mit ca. $89 \%$ den höchsten Anteil an richtigen Antworten auf. Bei der Frage zur Inflation ist mehr Variation in den richtigen Antworten über die Altersgruppen zu erkennen: Hier weist die Gruppe der 18- bis 34-jährigen mit $86 \%$ an richtigen Antworten den geringsten Anteil an richtigen Antworten auf, für die

\footnotetext{
3 Auf einen Verweis auf die Signifikanz wird bei den Ergebnissen über die Altersgruppen verzichtet, da es keine natürliche Referenzgruppe gibt. Gleiches gilt für die Ergebnisse über die Regionen und die Einkommens- sowie Vermögensquintile. Eine Tabelle mit den Signifikanzen ist von den Autoren auf Nachfrage erhältlich.
} 


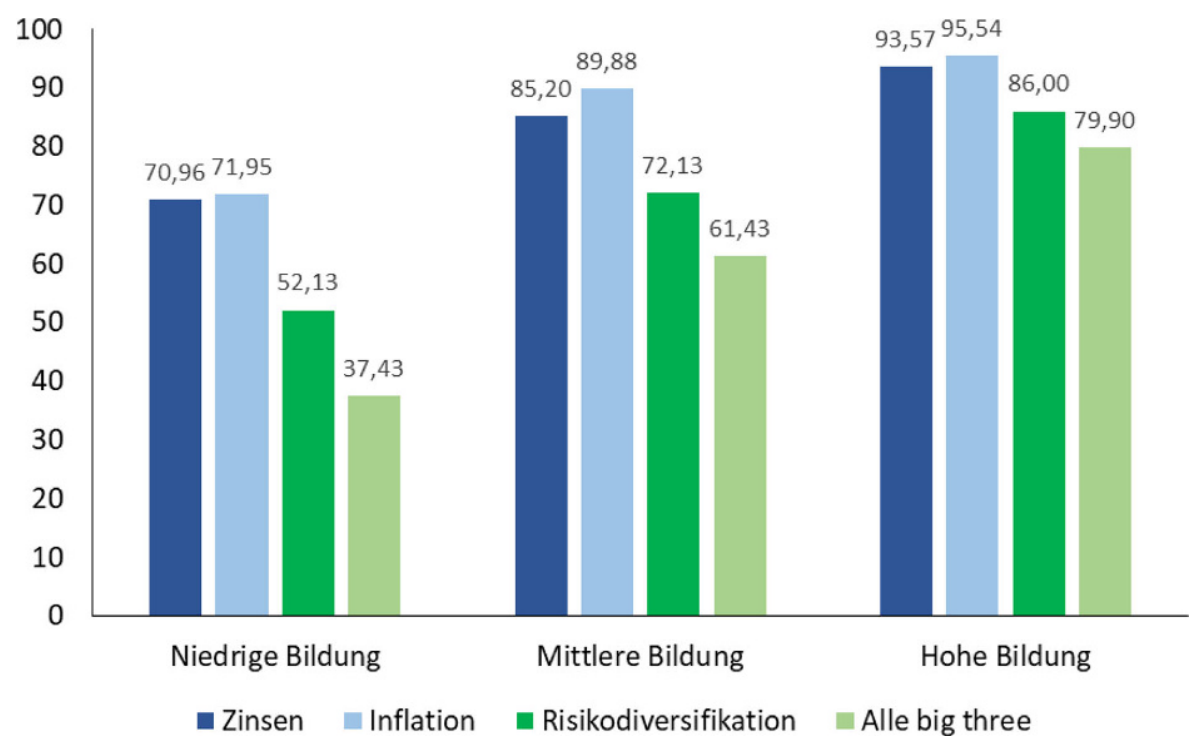

Abb. 3 Finanzkompetenzen nach Bildung. (Quelle: PHF 2010/2011, 2014, 2017. Eigene Berechnungen. Anmerkungen: Die Abbildung stellt den Anteil (in \%) an richtigen Antworten für die jeweilige Frage zur Finanzkompetenz, sowie den Anteil derer, die alle drei Fragen richtig beantwortet haben, dar. Die Einteilung in die Kategorien ist anhand der ,International Standard Classification for Education“ vorgenommen. Niedrige Bildung beinhaltet die Kategorien ISCED 0,1,2, mittlere Bildung beinhaltet die Kategorien ISCED 3,4,5 und hohe Bildung die Kategorien 6,7,8)

Gruppe der 55- bis 64-Jährigen sind mit etwa $91 \%$ die meisten richtigen Antworten erkennbar.

Bei der Frage zur Risikodiversifikation zeigt sich für die Altersgruppe der über 75-Jährigen mit 59\% der geringste, für die Altersgruppe der 45- bis 54-Jährigen mit $78 \%$ der höchste Anteil an richtigen Antworten. Insgesamt beantwortet die Altersgruppe der 35- bis 44-Jährigen mit 70\% den höchsten Anteil aller Fragen richtig. Den geringsten Anteil an richtigen Antworten weist die Altersgruppe der über 74-Jährigen mit lediglich $50 \%$ auf.

Ein weiteres Ergebnis der Literatur ist eine positive Korrelation zwischen formaler Bildung und finanziellen Grundkompetenzen (Lusardi und Mitchell 2011, 2014; Stolper und Walter 2017). Für Deutschland finden Lusardi und Mitchell (2014) basierend auf den SAVE-Daten für das Jahr 2009, dass nur 21,7\% derjenigen Personen, die lediglich über einen höchsten Bildungsabschluss der Sekundarstufe I verfügen, alle drei Fragen korrekt beantworten. Schon bei Personen, deren höchster Bildungsabschluss der Sekundarstufe II entspricht, ist dieser Anteil mit 51,6\% deutlich größer. Für Personen, die sogar über eine weiterführende Ausbildung oder einen Hochschulabschluss verfügen, fällt dieser Anteil mit über $70 \%$ nochmals deutlich höher aus.

Unklar ist in diesem Zusammenhang der Einfluss, der allein durch höhere kognitive Fähigkeiten entsteht. Denn da Personen mit einer höheren formalen Bildung auch häufig über höhere kognitive Fähigkeiten verfügen, ist dieser positive $\mathrm{Zu}$ sammenhang zwischen formaler Bildung und abgefragten Finanzkompetenzen nicht 


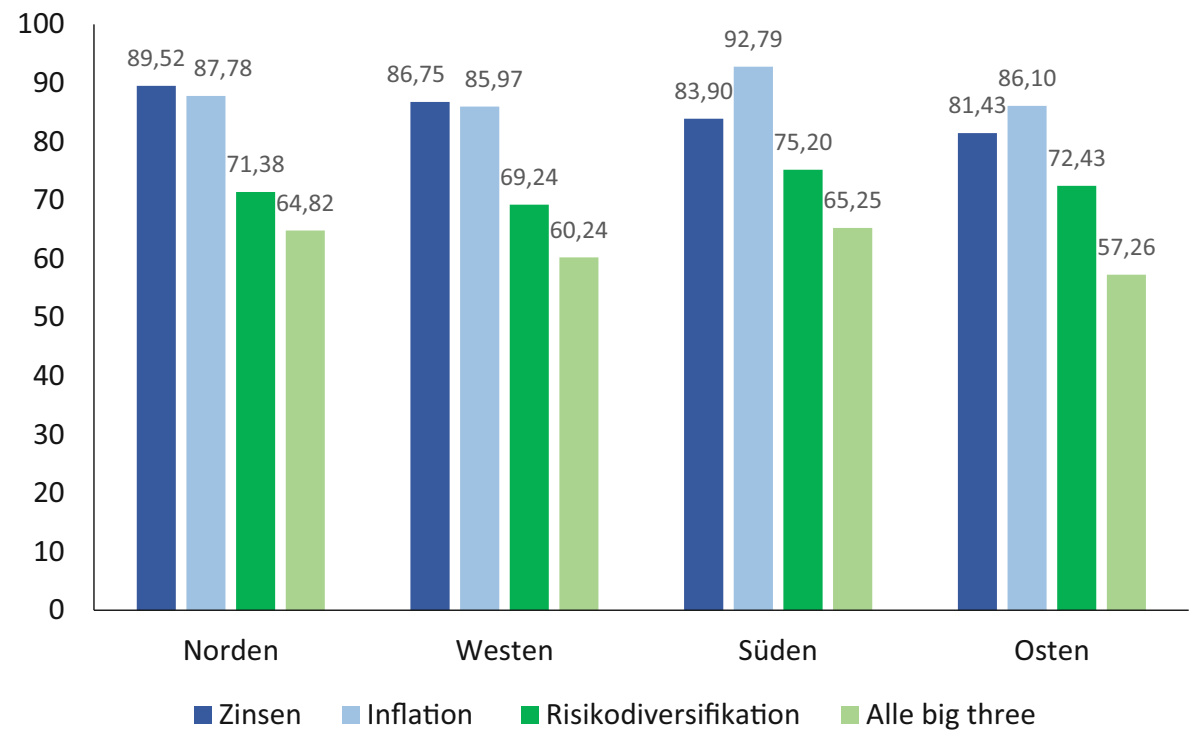

Abb. 4 Finanzkompetenzen nach Region. (Quelle: PHF 2010/2011, 2014, 2017. Eigene Berechnungen. Anmerkungen: Die Abbildung stellt den Anteil (in \%) an richtigen Antworten für die jeweilige Frage zur Finanzkompetenz, sowie den Anteil derer, die alle drei Fragen richtig beantwortet haben, dar. Die Region Norden umfasst die Bundesländer Bremen, Hamburg, Niedersachsen, und Schleswig-Holstein. Die Region Westen umfasst die Bundesländer Nordrhein-Westfalen, Rheinland-Pfalz sowie das Saarland. Die Region Süden umfasst die Bundesländer Baden-Württemberg, Bayern und Hessen. Die Region Osten umfasst die Bundesländer Berlin, Brandenburg, Mecklenburg-Vorpommern, Sachsen, Sachsen-Anhalt und Thüringen)

eindeutig auf die höhere Bildung zurückzuführen. Die Studienlage ist hier allerdings sehr spärlich. Jedoch zeigen Lusardi et al. (2010), dass formale Bildung, auch nachdem für kognitive Fähigkeiten kontrolliert wird, weiterhin einen relevanten Einfluss hat.

Anhand der PHF-Daten zeigt sich für Deutschland ebenfalls ein positiver Zusammenhang zwischen dem höchsten formalen Bildungsabschluss sowie der finanziellen Bildung. Wie in Abb. 3 zu erkennen ist, nimmt der Anteil an richtig beantworteten Fragen bei jeder Frage mit steigender Bildung zu. Unter den Personen mit dem geringsten formalen Bildungsstand beantworten lediglich $37 \%$ der Befragten alle drei Fragen richtig, bei Personen mit mittlerer Bildung ist dies bei $61 \%$ der Fall, und bei Personen mit hoher Bildung beantworten nahezu $80 \%$ der Befragten alle drei Fragen richtig. Alle in Abb. 3 dargestellten Differenzen sind statistisch signifikant auf dem $0,1 \%$-Niveau.

Neben den oben herausgestellten Beziehungen finden sich in der Literatur noch eine Reihe weiterer Zusammenhänge zwischen soziodemographischen Charakteristika und der finanziellen Bildung. So sind Unterschiede in der finanziellen Bildung über die Höhe des Einkommens und des Vermögens, für verschiedene Beschäftigungsarten, für Personen mit Migrationshintergrund und über verschiedene geografische Regionen zu beobachten.

Von besonderem Interesse für diese Arbeit ist insbesondere die Studie von $\mathrm{Bu}-$ cher-Koenen und Lamla-Dietrich (2018), die anhand der SAVE-Daten für 2009 


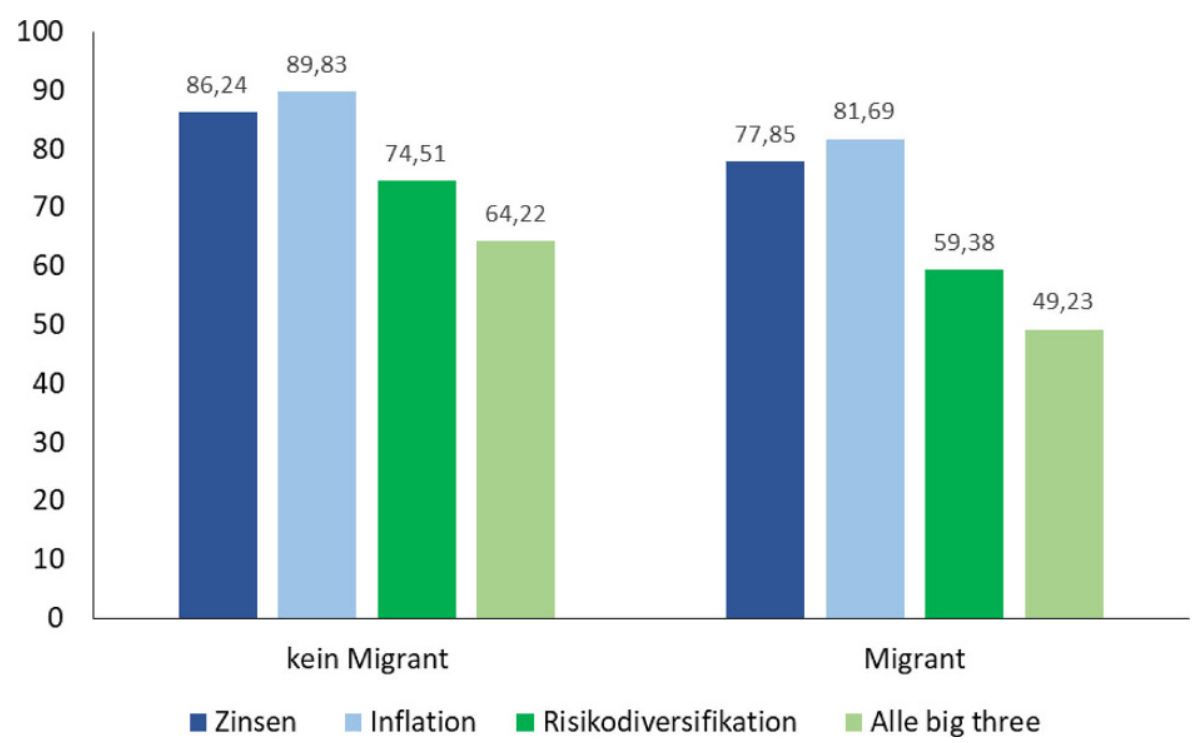

Abb. 5 Finanzkompetenzen nach Herkunft. (Quelle: PHF 2010/2011, 2014, 2017. Eigene Berechnungen. Anmerkungen: Die Abbildung stellt den Anteil (in \%) an richtigen Antworten für die jeweilige Frage zur Finanzkompetenz, sowie den Anteil derer die alle drei Fragen richtig beantwortet haben dar)

einen signifikanten Unterschied in der finanziellen Bildung zwischen Ost- und Westdeutschland ausweist, welcher auch über alle Bildungs-und Einkommensgruppen beobachtbar ist. Anhand einer Dekomposition zeigen die Autorinnen, dass die ermittelten Unterschiede in den Finanzkompetenzen zwischen in Ost- und Westdeutschland lebenden Personen nicht auf beobachtbare Charakteristika zurückzuführen sind. Ebenfalls für Deutschland zeigen Bucher-Koenen und Knebel (2021) basierend auf der jüngsten Welle des PHF, dass Ostdeutsche über eine geringere finanzielle Bildung verfügen als Westdeutsche.

Anhand der PHF-Daten können diese Ergebnisse ebenfalls für Deutschland gezeigt werden. Abb. 4 zeigt den Anteil der richtigen Antworten jeweils für Personen, die in den Stichprobenregionen Nord, West, Süd und Ost leben. Hier ist zu erkennen, dass ca. $65 \%$ der Befragten im Norden und Süden alle drei Fragen richtig beantworten. Im Westen liegt dieser Anteil mit $60 \%$ und im Osten mit $57 \%$ wesentlich niedriger. Das gleiche Muster ist ebenfalls für die Frage zur Inflation und zur Risikodiversifikation zu erkennen, bei denen Personen aus dem Westen und dem Osten ebenfalls seltener die Fragen richtig beantworten. ${ }^{4}$

Auch für Personen, die nicht in Deutschland geboren wurden, sind große Unterschiede hinsichtlich der finanziellen Bildung zu erkennen. In Deutschland geborene Personen beantworten alle Fragen häufiger richtig. Etwa $64 \%$ der Personen, die in Deutschland geboren sind, und $49 \%$ der Personen, die nicht in Deutschland geboren sind, beantworten alle ,big three“-Fragen richtig. Der in Abb. 5 dargestellte Unter-

\footnotetext{
${ }^{4}$ Das Sampling des Datensatzes zielt nicht auf regionale Repräsentativität ab. Daher werden nur recht weit gefasste Regionen miteinander verglichen.
} 


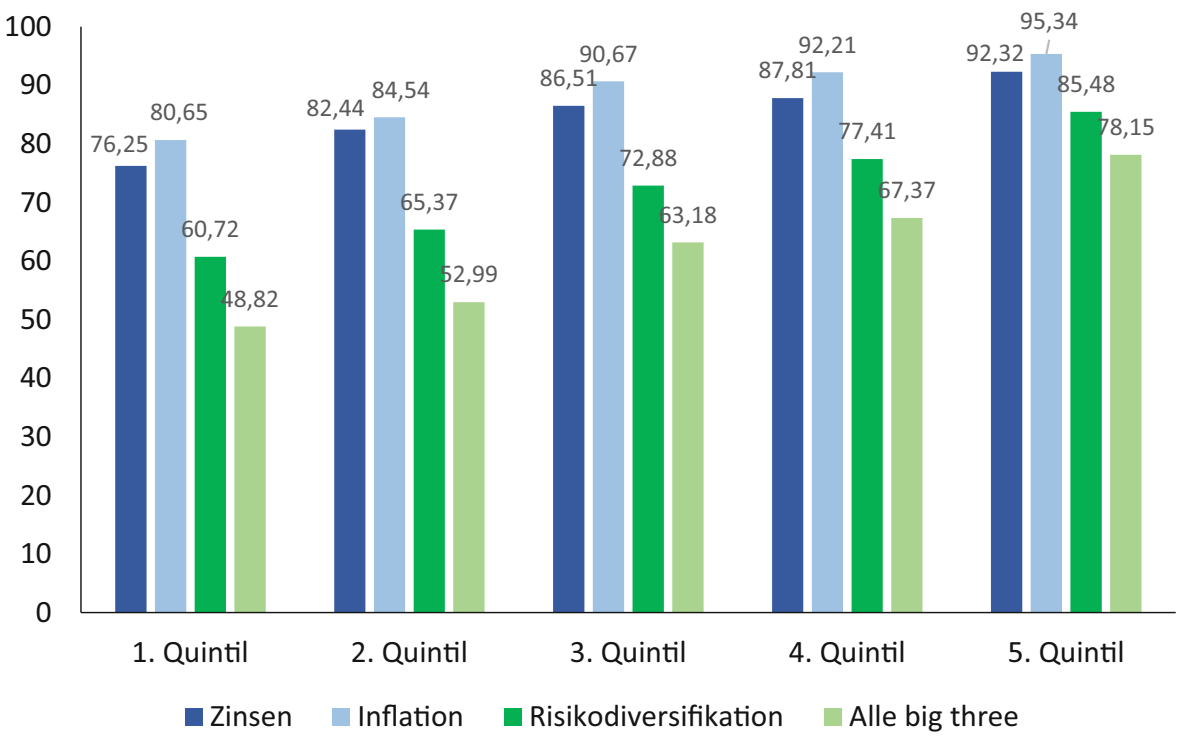

Abb. 6 Finanzkompetenzen nach Einkommensquintilen. (Quelle: PHF 2010/2011, 2014, 2017. Eigene Berechnungen. Anmerkungen: Die Abbildung stellt den Anteil (in \%) an richtigen Antworten für die jeweilige Frage zur Finanzkompetenz, sowie den Anteil derer, die alle drei Fragen richtig beantwortet haben, dar)

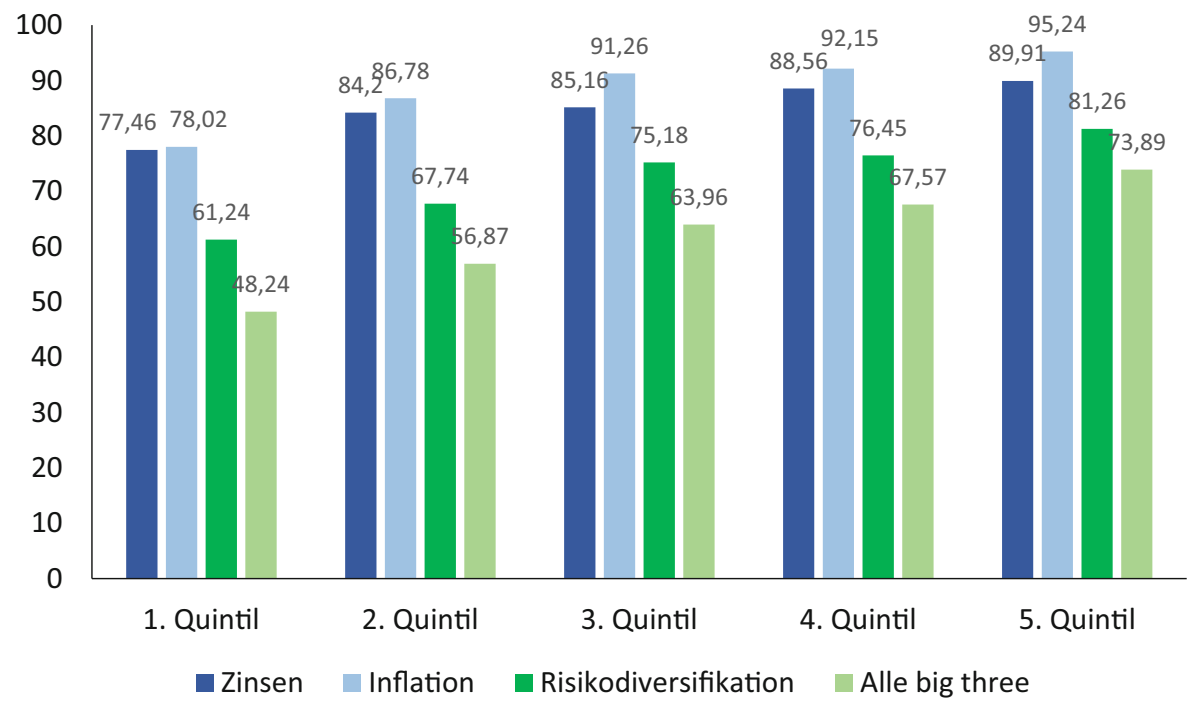

Abb. 7 Finanzkompetenzen nach Vermögensquintilen. (Quelle: PHF 2010/2011, 2014, 2017. Eigene Berechnungen. Anmerkungen: Die Abbildung stellt den Anteil (in \%) an richtigen Antworten für die jeweilige Frage zur Finanzkompetenz, sowie den Anteil derer, die alle drei Fragen richtig beantwortet haben, dar) 
schied, wie auch die Unterschiede bei den jeweils einzelnen Fragen, sind statistisch signifikant auf dem 0,1\%-Niveau.

Einen starken Zusammenhang mit der finanziellen Bildung weisen auch das Einkommen und das Vermögen der Haushalte auf. Die Abb. 6 und 7 stellen den Anteil an richtig beantworteten Fragen für die verschiedenen Quintile des Haushaltseinkommens und des Haushaltsvermögens dar. Die finanzielle Bildung nimmt sowohl mit steigendem Einkommen (Abb. 6) als auch mit steigendem Vermögen (Abb. 7) kontinuierlich zu. Die Daten des PHF zeigen, dass nur etwa $49 \%$ der Befragten im ersten Einkommensquintil alle ,big three“-Fragen richtig beantworten. Im letzten Quintil ist dieser Anteil um nahezu 30 Prozentpunkte höher und liegt bei etwa $78 \%$. Ein nahezu identisches Bild ergibt sich für die finanzielle Bildung über die Vermögensverteilung. Hier beantworten etwa $48 \%$ der Befragten im ersten Quintil und $74 \%$ im letzten Quintil alle ,big three“-Fragen richtig.

Tab. 2 Multivariater Zusammenhang zwischen den sozio-demographischen Charakteristika und der Finanzkompetenz. (Quelle: PHF 2010/2011, 2014, 2017, eigene Berechnungen)

\begin{tabular}{|c|c|c|c|c|}
\hline & Zinsen richtig & Inflation richtig & Risikodiversifikation richtig & Alle big three richtig \\
\hline \multirow[t]{2}{*}{$\overline{\text { Frau }}$} & $-0,020$ & $-0,029^{* * *}$ & $-0,036^{* *}$ & $-0,051^{* * * *}$ \\
\hline & $(0,011)$ & $(0,010)$ & $(0,013)$ & $(0,014)$ \\
\hline \multirow[t]{2}{*}{ Migrant } & $-0,070^{* * *}$ & $-0,046^{* *}$ & $-0,126^{* * *}$ & $-0,119^{* * *}$ \\
\hline & $(0,020)$ & $(0,018)$ & $(0,022)$ & $(0,022)$ \\
\hline \multicolumn{5}{|c|}{ Alter (ref.: 18-34) } \\
\hline \multirow[t]{2}{*}{$35-44$} & $-0,030$ & 0,004 & 0,035 & 0,012 \\
\hline & $(0,017)$ & $(0,017)$ & $(0,021)$ & $(0,024)$ \\
\hline \multirow[t]{2}{*}{$45-54$} & $-0,068^{* * *}$ & 0,004 & 0,008 & $-0,033$ \\
\hline & $(0,017)$ & $(0,016)$ & $(0,021)$ & $(0,022)$ \\
\hline \multirow[t]{2}{*}{$55-64$} & $-0,074^{* * *}$ & 0,021 & $-0,047^{*}$ & $-0,067^{* *}$ \\
\hline & $(0,018)$ & $(0,016)$ & $(0,022)$ & $(0,024)$ \\
\hline \multirow[t]{2}{*}{$65-74$} & $-0,095^{* * *}$ & 0,017 & $-0,086^{* * *}$ & $-0,115^{* * *}$ \\
\hline & $(0,019)$ & $(0,017)$ & $(0,023)$ & $(0,024)$ \\
\hline \multirow[t]{2}{*}{$75+$} & $-0,136^{* * *}$ & 0,004 & $-0,139^{* * *}$ & $-0,136^{* * *}$ \\
\hline & $(0,023)$ & $(0,019)$ & $(0,026)$ & $(0,027)$ \\
\hline \multicolumn{5}{|c|}{ Region (ref.: Westen) } \\
\hline \multirow[t]{2}{*}{ Norden } & 0,022 & 0,011 & 0,015 & 0,038 \\
\hline & $(0,015)$ & $(0,016)$ & $(0,021)$ & $(0,022)$ \\
\hline \multirow[t]{2}{*}{ Süden } & $-0,055^{* * *}$ & $0,043^{* * *}$ & 0,023 & 0,003 \\
\hline & $(0,014)$ & $(0,012)$ & $(0,016)$ & $(0,017)$ \\
\hline \multirow[t]{2}{*}{ Osten } & $-0,054^{* * *}$ & $-0,001$ & 0,035 & $-0,026$ \\
\hline & $(0,016)$ & $(0,015)$ & $(0,019)$ & $(0,020)$ \\
\hline \multicolumn{5}{|c|}{ Bildung (ref.: Niedrig) } \\
\hline \multirow[t]{2}{*}{ Mittel } & $0,091^{* * * *}$ & $0,136^{* * *}$ & $0,116^{* * *}$ & $0,152^{* * *}$ \\
\hline & $(0,021)$ & $(0,021)$ & $(0,024)$ & $(0,024)$ \\
\hline \multirow[t]{2}{*}{ Hoch } & $0,156^{* * *}$ & $0,173^{* * * *}$ & $0,216^{* * *}$ & $0,292^{* * *}$ \\
\hline & $(0,022)$ & $(0,022)$ & $(0,025)$ & $(0,026)$ \\
\hline
\end{tabular}


Tab. 2 (Fortsetzung)

\begin{tabular}{|c|c|c|c|c|}
\hline & Zinsen richtig & Inflation richtig & Risikodiversifikation richtig & Alle big three richtig \\
\hline \multicolumn{5}{|c|}{ Einkommen (ref.: Q1) } \\
\hline \multirow[t]{2}{*}{ Q2 } & $0,044^{*}$ & 0,003 & 0,012 & 0,003 \\
\hline & $(0,020)$ & $(0,018)$ & $(0,023)$ & $(0,023)$ \\
\hline \multirow[t]{2}{*}{ Q3 } & $0,063^{* * *}$ & $0,048^{* *}$ & $0,053^{*}$ & $0,066^{* *}$ \\
\hline & $(0,019)$ & $(0,017)$ & $(0,023)$ & $(0,024)$ \\
\hline \multirow[t]{2}{*}{ Q4 } & $0,049^{* *}$ & $0,044^{* *}$ & $0,057^{*}$ & $0,057^{*}$ \\
\hline & $(0,019)$ & $(0,017)$ & $(0,023)$ & $(0,025)$ \\
\hline \multirow[t]{2}{*}{ Q5 } & $0,070^{* * *}$ & $0,050^{* *}$ & $0,089^{* * * *}$ & $0,106^{* * *}$ \\
\hline & $(0,019)$ & $(0,017)$ & $(0,023)$ & $(0,025)$ \\
\hline \multicolumn{5}{|c|}{ Nettovermögen (ref.: Q1) } \\
\hline \multirow[t]{2}{*}{ Q2 } & $0,050^{* *}$ & $0,050^{* *}$ & 0,036 & $0,050^{*}$ \\
\hline & $(0,019)$ & $(0,018)$ & $(0,023)$ & $(0,023)$ \\
\hline \multirow[t]{2}{*}{ Q3 } & $0,044^{*}$ & $0,069^{* * *}$ & $0,069^{* *}$ & $0,076^{* *}$ \\
\hline & $(0,020)$ & $(0,018)$ & $(0,024)$ & $(0,025)$ \\
\hline \multirow[t]{2}{*}{ Q4 } & $0,076^{* * *}$ & $0,067^{* * *}$ & $0,081^{* * *}$ & $0,104^{* * *}$ \\
\hline & $(0,020)$ & $(0,017)$ & $(0,024)$ & $(0,025)$ \\
\hline \multirow[t]{2}{*}{ Q5 } & $0,079^{* * *}$ & $0,076^{* * *}$ & $0,103^{* * *}$ & $0,134^{* * * *}$ \\
\hline & $(0,020)$ & $(0,017)$ & $(0,024)$ & $(0,026)$ \\
\hline \multicolumn{2}{|c|}{ Konstante $0,754^{* * *}$} & $0,680^{* * *}$ & $0,548^{* * *}$ & $0,421^{* * *}$ \\
\hline & $(0,030)$ & $(0,029)$ & $(0,033)$ & $(0,034)$ \\
\hline$N$ & 12.700 & 12.700 & 12.700 & 12.700 \\
\hline$R^{2}$ & 0,070 & 0,079 & 0,090 & 0,107 \\
\hline
\end{tabular}

Anmerkungen: Robuste Standardfehler in Klammern (geclustert auf der Personenebene) $* p<0,05, * * p<0,01, * * * p<0,001$

\section{Multivariate Analyse}

In den bisherigen deskriptiven Analysen wurde lediglich ein bivariater Zusammenhang dargestellt, der es nicht erlaubt, die Wirkung dieser Faktoren gemeinsam zu betrachten. So dürfte ein Teil der untersuchten Faktoren untereinander eng zusammenhängen: Es ist z. B. wahrscheinlich, dass sich der Anteil der Personen mit Migrationshintergrund oder mit einem bestimmten Bildungsniveau regional unterscheidet. Ob ein Zusammenhang zwischen einer dieser Variablen und den finanziellen Grundkompetenzen tatsächlich statistisch signifikant ist, lässt sich daher sinnvollerweise nur in einer multivariaten Analyse betrachten.

Hierfür werden lineare Wahrscheinlichkeitsmodelle geschätzt, in denen die abhängige Variable den Wert 1 annimmt, falls die jeweilige Frage zur finanziellen Bildung richtig beantwortet wird. Darüber hinaus wird eine weitere Indikatorvariable gebildet, welche den Wert 1 annimmt, wenn alle drei Fragen gemeinsam richtig beantwortet werden. Die Variablen nehmen jeweils den Wert 0 an, wenn die entsprechenden Fragen falsch beantwortet werden. Als erklärende Variablen werden die zuvor beschriebenen Variablen Geschlecht, Migrationshintergrund, Alter, Region sowie die Einkommens- und Vermögensquintile des jeweiligen Haushalts, ausgedrückt 
in Indikator-Variablen, berücksichtigt. Zusätzlich werden noch Indikator-Variablen für die verschiedenen Wellen aufgenommen.

Die Ergebnisse dieser Schätzungen sind in Tab. 2 dargestellt. Dort finden die oben beschriebenen Zusammenhänge weitgehend ihre Bestätigung. So haben sowohl Frauen als auch Migranten über alle abhängigen Variablen eine geringere Ausprägung in der jeweiligen Finanzkompetenzvariable. Allerdings ist dieser Zusammenhang in der multivariaten Analyse weniger stark ausgeprägt als bei den oberen Analysen. So weisen Frauen, sobald für alle anderen Variablen kontrolliert wird, keine signifikant geringere Wahrscheinlichkeit auf, die Zinsfrage korrekt zu beantworten. Bei der Frage zur Inflation zeigen sich für Frauen nur noch eine um 2,9 Prozentpunkte und bei der Frage zur Risikodiversifikation eine um 3,6 Prozentpunkte geringere Wahrscheinlichkeit als Männer, die Frage korrekt zu beantworten. Die Wahrscheinlichkeit, alle drei Fragen richtig zu beantworten, wird für Frauen um 5,1 Prozentpunkte geringer geschätzt. Für Befragte mit Migrationshintergrund wird im Vergleich zu anderen Befragten die Wahrscheinlichkeit, die jeweilige Frage korrekt zu beantworten, um 7 (Zinsen), 4,6 (Inflation) und 12,6 (Risikodiversifikation) Prozentpunkte geringer eingeschätzt.

Ein eindeutiges Bild ist darüber hinaus beim Alter, der Bildung, dem Einkommen sowie dem Vermögen zu erkennen. Je höher der höchste Bildungsabschluss, desto höher ist den Schätzungen zufolge auch die Finanzkompetenz. Gleiches gilt für das Einkommen und das Vermögen. Beim Alter zeigt sich hier jedoch ein leicht verändertes Bild: So weist nun in fast jeder Kategorie die Gruppe der 18- bis 34jährigen die höchste Finanzkompetenz aus.

Bei der regionalen Herkunft der Personen ergibt sich ebenfalls ein leicht anderes Bild, sobald für weitere beobachtbare Charakteristika kontrolliert wird. So wird für Personen aus dem Süden und aus dem Osten im Vergleich zur Referenzkategorie (Westen) die Wahrscheinlichkeit als geringer eingeschätzt, die Zinsfrage korrekt zu beantworten. Darüber hinaus ist nur bei der Inflationsfrage ein signifikanter Unterschied zwischen im Westen lebenden Befragten und im Rest Deutschlands lebenden Befragten festzustellen. Hier weisen im Süden lebende Befragte eine um 4,3 Prozentpunkte höhere geschätzte Wahrscheinlichkeit auf, die Frage korrekt zu beantworten. ${ }^{5}$

Anzumerken ist in diesem Zusammenhang, dass die hier beschriebenen Ergebnisse lediglich als Korrelationen und nicht als Kausalitäten interpretiert werden dürfen. So ist insbesondere beim Einkommen und beim Vermögen davon auszugehen, dass nicht nur das Einkommen und das Vermögen die finanzielle Bildung beeinflussen, sondern dass umgekehrt auch Personen mit einer höheren finanziellen Bildung bessere Anlageentscheidungen treffen und somit tendenziell ein höheres Vermögen aufbauen als Personen mit einer geringeren finanziellen Bildung.

\footnotetext{
5 In weiteren Interaktionstests wurde der Frage nachgegangen, ob der Effekt für Frauen über die Höhe des Einkommens oder die Höhe des Vermögens variiert. Die Ergebnisse dieser Schätzungen zeigen, dass dies nicht der Fall ist, sie sind auf Nachfrage erhältlich.
} 


\section{Implikationen für die Wirtschafts- und Bildungspolitik}

Neben den oben dargestellten Ergebnissen sind insbesondere für wirtschafts- und bildungspolitischen Handlungsempfehlungen die Finanzkompetenzen von Heranwachsenden von Interesse. ${ }^{6}$ In diesem Lebensabschnitt stehen nicht nur ohnehin finanzielle Entscheidungen an, die über einen sehr langen Zeitraum wirken. Es zeichnet sich auch ab, dass die Gewichtung privater Vorsorge gegenüber staatlichen Renten in Zukunft weiter ansteigen wird, was zusätzlich ein höheres Maß an finanzieller Bildung bei Heranwachsenden wünschenswert macht. Lusardi et al. (2017) zeigen, dass sich 30 bis $40 \%$ der Unterschiede im Rentenvermögen auf Unterschiede in der finanziellen Bildung zurückführen lassen, weshalb auf Finanzkompetenz fokussierte Bildungsmaßnahmen diese Ungleichheit ein Stück weit reduzieren könnten.

Aus der Literatur wird ersichtlich, dass Maßnahmen zur Förderung der Finanzkompetenz sowohl bei Heranwachsenden als auch bei Erwachsenen erfolgreich sein können. Für Deutschland untersuchen Lührmann et al. (2015) und Lührmann et al. (2018) die Auswirkungen der Teilnahme an einer Finanzbildungsinitiative und zeigen, dass sich hierdurch das Interesse an Finanzzusammenhängen und das Verständnis für intertemporale Finanzentscheidungen erhöht. Unklarheit herrscht hingegen bezüglich der Wirksamkeit dieser Maßnahmen über längere Zeiträume. So ist die Motivation, das Erlernte anzuwenden, am höchsten, wenn die Entscheidungen kurz danach getroffen werden (Kaiser und Menkhoff 2017). Dies ist insbesondere in Bezug auf Schüler und Schülerinnen problematisch, da eine Vielzahl von Finanzentscheidungen erst einige Zeit nach dem Schulbesuch getroffen werden.

Darüber hinaus zeigt die Literatur, dass eine hohe Finanzkompetenz mit einer Reihe von sinnvollen Finanzentscheidungen einhergeht. So hat eine hohe Finanzkompetenz einen positiven Einfluss auf die Wahrscheinlichkeit, für die Rente zu planen, sowie auf die Höhe des Vermögens (Alessie et al. 2011; Arrondel et al. 2013; Bucher-Koenen und Knebel 2021; Bucher-Koenen und Lusardi 2011; Lusardi und Mitchell 2007, 2011; Sekita 2011; Van Rooij et al. 2011, 2012). Tendenziell werden fundiertere Anlageentscheidungen getroffen und Vermögenswerte in Krisenzeiten eher gehalten, anstatt mit Verlusten abgestoßen. Letzteres kann des Öfteren bei Personen mit geringer Finanzkompetenz beobachtet werden (Bucher-Koenen und Ziegelmeyer 2014). Außerdem nehmen Personen mit einer geringen Finanzkompetenz häufiger teurere Kredite in Anspruch, sind häufiger verschuldet und benutzen Kreditkarten mit höheren Gebühren (Lusardi und Tufano 2015).

Wie unter anderem in Studien von Knoll und Houts (2012) sowie Nicolini und Haupt (2019) gezeigt wird, sind die „big three“ jedoch nicht ausreichend in der Lage, die volle Bandbreite der Finanzkompetenz zu erfassen. Da ein Großteil der Forschung, auch mangels gegebener Alternativen, bisher lediglich einen Zusammenhang basierend auf den „big three“ herstellt, besteht noch ein großer Bedarf darin, den Bezug zwischen Finanzkompetenz und den Finanzentscheidungen genauer zu analysieren (Nicolini und Haupt 2019). Hierbei erscheint insbesondere eine Erweiterung der Datenbasis zu Finanzkompetenzen über die „big three“ hinaus bedeutsam.

\footnotetext{
${ }^{6}$ Siehe z. B. Driva et al. (2016) und Erner et al. (2016) für Ergebnisse zu den Finanzkompetenzen bei Schulkindern.
} 
Eine erweiterte Datenbasis würde zudem die Möglichkeit eröffnen, bisher nicht beantwortete Forschungsfragen zu analysieren. So würden Datensätzen, die größere Fallzahlen aufweisen und sich zudem nicht nur auf eine Person des Haushalts beziehen, eine tiefergehende Untersuchung der finanziellen Kompetenzen verschiedener Teile der Bevölkerung ermöglichen, bspw. von Frauen in unterschiedlichen Altersgruppen oder mit bzw. ohne Migrationshintergrund. Zudem wäre von großem Interesse, den Bezug zwischen Finanzkompetenz und finanziellen Verhaltensweisen bzw. entsprechenden Entscheidungen zu analysieren.

Vor dem Hintergrund der Ergebnisse unserer Studie sowie der Literatur erscheint es sinnvoll, die Finanzkompetenzen sowohl bei heranwachsenden, wie auch bei erwachsenen Individuen weiter zu stärken. Erste Schritte in diese Richtung wurden in manchen Bundesländern mit der Einbindung des Schulfachs Wirtschaft in den Lehrplan bereits erfolgreich eingeleitet. Die Länder setzen dieses Vorhaben jedoch sehr unterschiedlich um. So haben einige Bundesländer das Fach bereits in den Lehrplan integriert, wohingegen es in anderen Ländern als Querschnittsthema betrachtet wird. Dort werden zwar Inhalte wie Zinsen und Inflation im Fach Mathematik, oder Themen wie Globalisierung in Fächern wie Sozialwissenschaften oder Geographie unterrichtet, allerdings wird bei diesen Themen nicht immer ein wirtschaftlicher Bezug hergestellt und die Zusammenhänge nicht als Gesamtes betrachtet (Bundeszentrale für politische Bildung 2019).

Daher erscheint ein systematischeres Vorgehen bei der Einführung bzw. dem Ausbau des Schulfachs Wirtschaft sehr sinnvoll. Darüber hinaus ist das Angebot an Studiengängen im Bereich der Wirtschaftswissenschaften für angehende Lehrer und Lehrerinnen sehr gering, was zur Folge hat, dass die wenigsten Lehrer an den Schulen mit diesen Themen vertraut sind und dies dementsprechend als Fachfremde unterrichten. Dies erscheint aufgrund der Komplexität der wirtschaftlichen Themen wenig sinnvoll. Es bietet sich daher an, das Angebot eigenständiger Studiengänge im Bereich Wirtschaft für die angehenden Lehrer und Lehrerinnen auszubauen. Ein systematischer Ausbau des Schulfachs Wirtschaft scheitert dabei unter anderem an Bedenken, dass andere Bildungsinhalte dadurch zu kurz kommen könnten (siehe z. B. Deutscher Gewerkschaftsbund NRW 2018). Zudem erscheint die diesbezügliche Diskussion politisch bzw. ideologisch aufgeheizt, was der konstruktiven Erarbeitung einer Strategie zur Verbesserung der Finanzkompetenzen wenig zuträglich ist (Bundeszentrale für politische Bildung 2019; Deutscher Gewerkschaftsbund 2012; Engartner 2013).

Bezüglich der geringen finanziellen Bildung von Frauen und Migranten scheint es außerdem sinnvoll, ein größeres Angebot an Bildungsmöglichkeiten für Erwachsene zur Verfügung zu stellen. So könnten zum Beispiel in denjenigen Lebenssituationen, nach denen üblicherweise größere Finanzentscheidungen getroffen werden, z. B. nach dem Schul- oder Studienabschluss oder der Geburt eines Kindes, mehr Informationen zum Thema Finanzen und ein Verweis auf bereits existierende Beratungsangebote zur Verfügung gestellt werden.

Eine weitere Maßnahme könnte darin bestehen, die Transparenz und Verständlichkeit von Finanzprodukten zu erhöhen. Hierbei wäre eine Möglichkeit, detailliertes, leicht verständliches Informationsmaterial bei der Beratung zu diesen Finanzinstrumenten verpflichtend bereitzustellen. Ein erster Schritt in diese Richtung wurde 
als Reaktion auf die Finanzkrise 2008 unternommen, als im Rahmen des Wertpapierhandelsgesetzes (WpHG) die Offenlegung von Risikoeinschätzungen und die ex-ante Kosteninformationen seitens des Anlageberaters gegenüber von Kleinanlegern verpflichtend gemacht wurde (Bundesanstalt für Finanzdienstleistungsaufsicht 2018).

Handlungsbedarf im Bereich der Finanzprodukte für die Altersvorsorge besteht insbesondere bei der Riester-Rente, da diese sehr komplex gestaltet ist. Für Interessenten ist es daher häufig schwer, die eigene Förderfähigkeit oder die Höhe des Mindesteigenbetrags einzuschätzen, um die volle Förderung zu erhalten. Hierdurch kommt es häufig zu Rückforderungen der Zulagen, was zu einem Vorsorgehemmnis werden kann. Darüber hinaus werden die hohe Marktintransparenz und zu hohe Kosten bei den Verträgen kritisiert (Börsch-Supan et al. 2016; Lueg und Schwark 2019), was durch entsprechende Gesetzgebung verhindert werden könnte.

\section{Zusammenfassung}

Die vorliegende Studie zeigt, dass die finanzielle Bildung in Deutschland im internationalen Vergleich gut ausgeprägt zu sein scheint. Etwas mehr als $60 \%$ der Befragten können alle drei Standardfragen zur Messung der finanziellen Kompetenzen richtig beantworten. Diese Fragen messen das Wissen der Studienteilnehmer in den Bereichen Zinsen, Inflation sowie Risikodiversifikation. Die Ergebnisse zeigen aber auch, dass es noch erhebliches Potenzial für Verbesserungen gibt. Zwar liegen die deutschen Haushalte mit ihrem Finanzwissen im oberen Mittelfeld, jedoch wird insbesondere vor dem Hintergrund der zu erwartenden Rentenlücke und der lang andauernden Niedrigzinsphase ein anderes Anlageverhalten notwendig werden. Darüber hinaus ist ein internationaler Vergleich der finanziellen Kompetenzen nur eingeschränkt möglich. Aus diesem Grund und weil auf Grundlage der vorliegenden Datenbasis die Möglichkeiten, ein tieferes Verständnis für die finanziellen Kompetenzen zu entwickeln, kaum gegeben sind, herrscht weiterhin ein großer Bedarf nach weiteren Ansätzen, die finanziellen Kompetenzen zu messen.

Die Studie dokumentiert darüber hinaus eine große Heterogenität in den Finanzkompetenzen über verschiedene sozio-demografische Charakteristika hinweg. In den deskriptiven Analysen ist ein großer Unterschied in den Finanzkompetenzen zwischen Männern und Frauen, sowie zwischen Personen mit bzw. ohne Migrationshintergrund festzustellen. Diese Ergebnisse dürfen jedoch nicht als kausale Zusammenhänge interpretiert werden. Hier sind weitere Studien notwendig, um Risikogruppen noch präziser zu identifizieren.

Darüber hinaus konnte anhand der Literatur gezeigt werden, dass Maßnahmen zur Förderung der Finanzkompetenz sowohl bei Erwachsenen als auch bei Kindern erfolgreich sein können. In der Regel wird Wissen jedoch nur angewandt, wenn es kurz vorher erlernt wurde. Ob sich bei den Maßnahmen für sehr junge Personen langfristige Effekte einstellen, ist derzeit noch nicht klar. Konkrete wirtschaftspolitische Maßnahmen zur Verbesserung der Finanzkompetenz, deren Einsatz erwogen werden sollte, sind die verstärkte Übernahme von entsprechenden Inhalten in die Lehrpläne von Schulen, eine verbesserte Ausbildung von Lehrern in diesem Bereich, ein bes- 
seres Angebot von Bildungs- und Informationsangeboten für Erwachsene und eine verbesserte Transparenz im Bereich von Finanz- und Versicherungsprodukten.

\section{Anhang}

Die Fragen zur finanziellen Bildung in den PHF-Daten, diese sind in den drei vorliegenden Wellen identisch (PHF Codebook 2010, 2014, 2017):

1. Angenommen, Sie haben $100 €$ Guthaben auf ihrem Sparkonto. Dieses Guthaben wird mit $2 \%$ pro Jahr verzinst, und Sie lassen es 5 Jahre auf diesem Konto. Was meinen Sie: Wie hoch wird ihr Guthaben nach 5 Jahren sein?
a. Höher als $102 €$
b. Genau $102 €$
c. Niedriger als $102 €$
d. Weiß nicht
e. Keine Antwort

2. Angenommen, die Verzinsung Ihres Sparkontos beträgt $1 \%$ pro Jahr und die Inflationsrate beträgt $2 \%$ pro Jahr. Was glauben Sie: Werden Sie nach einem Jahr mit dem Guthaben des Sparkontos genauso viel, mehr oder weniger als heute kaufen können?
a. Mehr
b. Genauso viel
c. Weniger als heute
d. Weiß nicht
e. Keine Antwort

3. Stimmen Sie der folgenden Aussage zu: „Die Anlage in Aktien eines einzelnen Unternehmens ist weniger riskant als die Anlage in einem Fonds mit Aktien ähnlicher Unternehmen“?
a. Stimme zu
b. Stimme nicht zu
c. Weiß nicht
d. Keine Antwort

Danksagung Wir danken Friederike Hertweck und einer/einem anonymen Gutachter/_in für hilfreiche Hinweise und dem Ministerium für Kultur und Wissenschaft des Landes NRW sowie dem Bundesministerium für Wirtschaft für ihre Unterstützung der Forschungsgruppe Bildung am RWI im Rahmen des „Zukunftsprogramm RWI 2020“.

Funding Open Access funding enabled and organized by Projekt DEAL.

Open Access Dieser Artikel wird unter der Creative Commons Namensnennung 4.0 International Lizenz veröffentlicht, welche die Nutzung, Vervielfältigung, Bearbeitung, Verbreitung und Wiedergabe in jeglichem Medium und Format erlaubt, sofern Sie den/die ursprünglichen Autor(en) und die Quelle ordnungsgemäß nennen, einen Link zur Creative Commons Lizenz beifügen und angeben, ob Änderungen vorgenommen wurden.

Die in diesem Artikel enthaltenen Bilder und sonstiges Drittmaterial unterliegen ebenfalls der genannten Creative Commons Lizenz, sofern sich aus der Abbildungslegende nichts anderes ergibt. Sofern das betref- 
fende Material nicht unter der genannten Creative Commons Lizenz steht und die betreffende Handlung nicht nach gesetzlichen Vorschriften erlaubt ist, ist für die oben aufgeführten Weiterverwendungen des Materials die Einwilligung des jeweiligen Rechteinhabers einzuholen.

Weitere Details zur Lizenz entnehmen Sie bitte der Lizenzinformation auf http://creativecommons.org/ licenses/by/4.0/deed.de.

\section{Literatur}

Alessie, R., Van Rooij, M., \& Lusardi, A. (2011). Financial literacy and retirement preparation in the Netherlands. Journal of Pension Economics \& Finance, 10(4), 527.

Altmann, K., Bernard, R., Le Blanc, J., Gabor-Toth, E., Hebbat, M., Kothmayr, L., Schmidt, T., Tzamourani, P., Werner, D., \& Zhu, J. (2020). The Panel on Household Finances (PHF)—Microdata on household wealth in Germany. German Economic Review, 21(3), 373-400.

Agnew, J.R., Bateman, H. \& Thorp, S. (2013). Financial literacy and retirement planning in Australia. Numeracy, 6(2), 1-25.

Arrondel, L., Debbich, M., \& Savignac, F. (2013). Financial literacy and financial planning in France. Numeracy, 6(2), 8.

Bannier, C.E., \& Neubert, M. (2016). Gender differences in financial risk taking: the role of financial literacy and risk tolerance. Economics Letters, 145, 130-135.

Bianchi, M. (2018). Financial literacy and portfolio dynamics. The Journal of Finance, 73(2), 831-859.

Börsch-Supan, A., Bucher-Koenen, T., Goll, N., \& Maier, C. (2016). 15 Jahre Riester - eine Bilanz. Arbeitspapier 12/2016. : Munich Center for the Economics of Aging.

Börsch-Supan, A., Coppola, M., Essig, L., Eymann, A., \& Schunk, D. (2008). The German SAVE study: design and results

Brown, M. \& Graf, R. (2013). Financial literacy and retirement planning in Switzerland. Numeracy, 6(2), Article 6.

Bucher-Koenen, T., \& Knebel, C. (2021). Finanzwissen und Finanzbildung in Deutschland - Was wissen wir eigentlich? ZEW Discussion Paper 21-016.

Bucher-Koenen, T., \& Lamla-Dietrich, B. (2018). The long shadow of socialism: puzzling evidence on East-West German differences in financial literacy. Economic Notes: Review of Banking, Finance and Monetary Economics, 47(2/3), 413-438.

Bucher-Koenen, T., \& Lusardi, A. (2011). Financial literacy and retirement planning in Germany. Journal of Pension Economics \& Finance, 10(4), 565-584.

Bucher-Koenen, T., \& Ziegelmeyer, M. (2014). Once burned, twice shy? Financial literacy and wealth losses during the financial crisis. Review of Finance, 18(6), 2215-2246.

Bucher-Koenen, T., Lusardi, A., Alessie, R., \& Van Rooij, M. (2017). How financially literate are women? An overview and new insights. Journal of Consumer Affairs, 51(2), 255-283.

Bundesanstalt für Finanzdienstleistungsaufsicht (2018). „Nachhaltige Finanzwirtschaft.“ BaFin Journal Mai 2018: https://www.bafin.de/dok/10888904. Zugegriffen: 4. Aug. 2021.

Bundeszentrale für politische Bildung (Hrsg.). (2019). Brauchen wir ein eigenes Unterrichtsfach Wirtschaft? https://www.bpb.de/gesellschaft/bildung/politische-bildung/301282/fach-wirtschaft. Zugegriffen: 4. Aug. 2021.

Christelis, D., Jappelli, T., \& Padula, M. (2010). Cognitive abilities and portfolio choice. European Economic Review, 54(1), 18-38.

Deutscher Gewerkschaftsbund (Hrsg.). (2012). Wirtschaft in der Schule - Was sollen unsere Kinder Lernen? DGB Positionspapier. https://www.dgb.de/themen/++co++dbb55b44-0ccd-11e4-b73a52540023ef1a. Zugegriffen: 4. Aug. 2021.

Deutscher Gewerkschaftsbund NRW (Hrsg.). (2018). Schulfach Wirtschaft geht an gesellschaftlicher Realität vorbei. https://nrw.dgb.de/presse-und-social-media/++co++da7c36bc-f17a-11e8-b7f652540088cada. Zugegriffen: 4. Aug. 2021.

Driva, A., Lührmann, M., \& Winter, J. (2016). Gender differences and stereotypes in financial literacy: off to an early start. Economics Letters, 146, 143-146.

Engartner, T. (2013). Das Fach „Wirtschaft“ als Fach der Wirtschaft? Einige ausgewählte Aspekte vergangener und gegenwärtiger Debatten. GWP-Gesellschaft. Wirtschaft. Politik, 62(3), 29-30.

Erner, C., Goedde-Menke, M., \& Oberste, M. (2016). Financial literacy of high school students: evidence from Germany. The Journal of Economic Education, 47(2), 95-105. 
Goetzmann, W.N., \& Kumar, A. (2008). Equity portfolio diversification. Review of Finance, 12(3), 433-463.

Grinblatt, M., Keloharju, M., \& Linnainmaa, J. (2011). IQ and stock market participation. The Journal of Finance, 66(6), 2121-2164.

Hastings, J.S., Madrian, B.C., \& Skimmyhorn, W.L. (2013). Financial literacy, financial education, and economic outcomes. Annual Review of Economics, 5(1), 347-373.

Hsu, J.W. (2016). Aging and strategic learning: The impact of spousal incentives on financial literacy. Journal of Human Resources, 51(4), 1036-1067.

Kaiser, T., \& Menkhoff, L. (2017). Does financial education impact financial literacy and financial behavior, and if so, when? The World Bank Economic Review, 31(3), 611-630.

Knoll, M. A., \& Houts, C.R. (2012). The financial knowledge scale: an application of item response theory to the assessment of financial literacy. Journal of Consumer Affairs, 46(3), 381-410.

Lueg, T., \& Schwark, P. (2019). Staatsfonds in der individuellen Alterssicherung - kein Modell für Deutschland. Vierteljahrshefte zur Wirtschaftsforschung, 88(1), 125-140.

Lührmann, M., Serra-Garcia, M., \& Winter, J. (2015). Teaching teenagers in finance: does it work? Journal of Banking \& Finance, 54, 160-174.

Lührmann, M., Serra-Garcia, M., \& Winter, J. (2018). The impact of financial education on adolescents' intertemporal choices. American Economic Journal: Economic Policy, 10(3), 309-332.

Lusardi, A. (2019). Financial literacy and the need for financial education: evidence and implications. Swiss Journal of Economics and Statistics, 155(1), 1-8.

Lusardi, A., \& Mitchell, O.S. (2007). Baby boomer retirement security: the roles of planning, financial literacy, and housing wealth. Journal of Monetary Economics, 54(1), 205-224.

Lusardi, A., \& Mitchell, O.S. (2008). Planning and financial literacy: how do women fare? American Economic Review, 98(2), 413-417.

Lusardi, A., \& Mitchell, O.S. (2011). Financial literacy around the world: an overview. Journal of Pension Economics and Finance, 10(4), 497-508.

Lusardi, A., \& Mitchell, O.S. (2014). The economic importance of financial literacy: theory and evidence. Journal of Economic Literature, 52(1), 5-44.

Lusardi, A., \& Tufano, P. (2015). Debt literacy, financial experiences, and overindebtedness. Journal of Pension Economics \& Finance, 14(4), 332-368.

Lusardi, A., Michaud, P.-C., \& Mitchell, O. S. (2017). Optimal financial knowledge and wealth inequality. Journal of Political Economy, 125(2), 431-477.

Lusardi, A., Mitchell, O.S., \& Curto, V. (2010). Financial literacy among the young. Journal of Consumer Affairs, 44(2), 358-380.

Nicolini, G., \& Haupt, M. (2019). The assessment of financial literacy: new evidence from Europe. International Journal of Financial Studies, 7(3), 54.

Nicolosi, G., Peng, L., \& Zhu, N. (2009). Do individual investors learn from their trading experience? Journal of Financial Markets, 12(2), 317-336.

OECD (2020). OECD/INFE 2020 international survey of adult financial literacy. https://www.oecd. org/financial/education/launchoftheoecdinfeglobalfinancialliteracysurveyreport.htm. Zugegriffen: 4. Aug. 2021.

PHF Codebook (Hrsg.). (2010). Fragenprogramm Private Haushalte und ihre Finanzen. https://www. bundesbank.de/resource/blob/617368/b0ee105da2e3919f38ddb69a1bd73da3/mL/phf-codebook-dedata.pdf. Zugegriffen: 4. Aug. 2021.

PHF Codebook (Hrsg.). (2014). Fragenprogramm Private Haushalte und ihre Finanzen. https://www. bundesbank.de/resource/blob/617344/62a1001a29335589d411940282c7ce79/mL/phf-codebookwave2-de-data.pdf. Zugegriffen: 4. Aug. 2021.

PHF Codebook (Hrsg.). (2017). Fragenprogramm Private Haushalte und ihre Finanzen. https://www. bundesbank.de/resource/blob/798118/117e900b9583f5380e846cc7d1e42cff/mL/phf-codebook-wav e3-de-data.pdf. Zugegriffen: 4. Aug. 2021.

Richter, D., \& Schupp, J. (2015). The soep innovation sample (soep is). Schmollers Jahrbuch: Journal of Applied Social Science Studies/Zeitschrift für Wirtschafts- und Sozialwissenschaften, 135(3), 389-400.

Schmidt, T., \& Tzamourani, P. (2017). Zur finanziellen Bildung der privaten Haushalte in Deutschland: Ausgewählte Ergebnisse aus der Studie „Private Haushalte und ihre Finanzen (PHF)“. Vierteljahrshefte zur Wirtschaftsforschung, 86(4), 31-49.

Sekita, S. (2011). Financial literacy and retirement planning in Japan. Journal of Pension Economics and Finance, 10(4), 637-656. 
Seru, A., Shumway, T., \& Stoffman, N. (2010). Learning by trading. The Review of Financial Studies, 23(2), 705-739.

Stolper, O. A., \& Walter, A. (2017). Financial literacy, financial advice, and financial behavior. Journal of Business Economics, 87(5), 581-643.

Van Rooij, M., Lusardi, A., \& Alessie, R. (2011). Financial literacy and stock market participation. Journal of Financial Economics, 101(2), 449-472.

Van Rooij, M. C., Lusardi, A., \& Alessie, R. J. (2012). Financial literacy, retirement planning and household wealth. The Economic Journal, 122(560), 449-478. 